\title{
The relationship between body mass index and uric acid: a study on Japanese adult twins
}

\author{
Kentaro Tanaka ${ }^{1} \cdot$ Soshiro Ogata ${ }^{2} \cdot$ Haruka Tanaka ${ }^{2,3} \cdot$ Kayoko Omura $^{3}$. \\ Chika Honda $^{4} \cdot$ Osaka Twin Research Group $\cdot$ Kazuo Hayakawa ${ }^{3}$
}

Received: 20 March 2015/Accepted: 24 May 2015/Published online: 3 June 2015

(C) The Japanese Society for Hygiene 2015

\begin{abstract}
Objectives The present study aimed to investigate the association between body mass index (BMI) and uric acid (UA) using the twin study methodology to adjust for genetic factors.

Methods The association between BMI and UA was investigated in a cross-sectional study using data from both monozygotic and dizygotic twins registered at the Osaka University Center for Twin Research and the Osaka University Graduate School of Medicine. From January 2011 to March 2014, 422 individuals participated in the health examination. We measured height, weight, age, BMI, lifestyle habits (Breslow's Health Practice Index), serum UA, and serum creatinine. To investigate the association between UA and BMI with adjustment for the clustering of a twin within a pair, individual-level analyses were performed using generalized linear mixed models (GLMMs). To investigate an association with adjustment
\end{abstract}

Osaka Twin Research Group (Kazuo Hayakawa, Yoshinori Iwatani, Jun Hatazawa, Shiro Yorifuji, Mikio Watanabe, Chika Honda, and Kayoko Omura, Center for Twin Research, Osaka University Graduate School of Medicine).

Kentaro Tanaka

ktanaka@gifu-u.ac.jp

1 Department of Community Health Nursing, Gifu University School of Medicine, 1-1 Yanagido, Gifu City, Gifu 501-1194, Japan

2 Department of Health Promotion Science, Osaka University Graduate School of Medicine, Osaka, Japan

3 Mie Prefectural College of Nursing, Mie, Japan

4 Center for Twin Research, Osaka University Graduate School of Medicine, Osaka, Japan for genetic and family environmental factors, twin-pair difference values analyses were performed.

Results In all analysis, BMI was associated with UA in men and women. Using the GLMMs, standardized regression coefficients were 0.194 (95\% confidence interval: $0.016-0.373)$ in men and 0.186 (95 \% confidence interval: 0.071-0.302) in women. Considering twin-pair difference value analyses, standardized regression coefficients were 0.333 (95\% confidence interval: $0.072-0.594$ ) in men and 0.314 (95\% confidence interval: $0.151-0.477$ ) in women. Conclusions The present study shows that BMI was significantly associated with UA, after adjusting for both genetic and familial environment factors in both men and women.

Keywords Twin study - Genetics - Environment · Uric acid · Body mass index

\section{Introduction}

The prevalence of high uric acid (UA) levels has increased worldwide in both men and women [1-3]. In Japan, it has been shown that UA levels in male adolescents have increased during the last 12 years, and there has been an increased incidence of hyperuricemia reported along with increased body mass index (BMI) [4]. In addition, UA is associated with an increased risk of developing cardiovascular disease, particularly heart failure and ischemic heart disease [5, 6]. Furthermore, several previous studies have indicated that people have developed high UA because of genetic factors, alcohol, obesity, and weight gain [7].

Other epidemiological studies have suggested that a high BMI triggers risk factors for lifestyle-related diseases 
such as type 2 diabetes, cardiovascular diseases, cancers, and high UA $[8,9]$. In fact, a study by De Oliveira et al. has shown that individuals with BMI $\geq 25 \mathrm{~kg} / \mathrm{m}^{2}$ showed greater chances of high UA levels [odds ratio $(\mathrm{OR})=2.28$ (1.13-4.6)] [10]. Therefore, this study suggests that elevated BMI levels are closely related to high UA levels, and in order to prevent high UA, one should pay attention to BMI level changes.

Current research indicates that UA and BMI are influenced by genetic factors. The National Heart, Lung, and Blood Institute (NHLBI) twin study has demonstrated high heritability for UA [11]. In addition, specific genes which relate to UA, particularly genes e.g., SLC2A9, ABCG2, $S L C 22 A 12$, and $M A F$, which encode for proteins involved in renal urate excretion, have been reported as being associated with genetic susceptibility to hyperuricemia [12, 13]. Additionally, a systematic review of genetic studies on BMI has reported a strong genetic influence, and a genome-wide association study in Japan identified two novel BMI-associated loci e.g., CDKAL1 and KLF9 [14, 15]. However, the association between UA and BMI may be confounded by genetic factors, since the previous epidemiological studies did not adjust for genetic factors. Therefore, the present study aimed to investigate the association between BMI and UA using the twin study methodology in order to adjust for both genetic and familial environmental factors.

\section{Methods}

\section{Study participants}

The association between BMI and UA was investigated using a cross-sectional study and exploited data from both monozygotic (MZ) and dizygotic (DZ) twins registered at the Osaka University Center for Twin Research (OUCTR) and the Osaka University Graduate School of Medicine. From January 2011 to March 2014, 422 individuals participated in the health examination. The project aims to preserve and store biological resources and data to databases and bio-banking resources [16]. All registrants were recruited through newspaper advertisements, posters, leaflets, public lectures, and symposia. We sent invitation letters to registered twins (aged 20 years or older) living in Japan. Only twins who were both alive and consented to participate in the study were invited for comprehensive medical examination at Osaka University [16]. The eligibility criteria excluded twin participants who were not of the same sex. Zygosity was confirmed using the 15 loci of short tandem repeat (STR) markers, a well-recognized method for true ascertainment of twins [17]. An entirely concordant pair with the aforementioned STRs was diagnosed as a MZ twin pair. All other pairs were designated as DZ [16]. The present study was approved by the Institutional Review Board for Clinical Research at Osaka University Hospital and the Research Ethics Committee of Osaka University.

\section{Measurement of biochemical and physiological markers}

\section{BMI, UA, Creatinine}

BMI was calculated as weight $/ \mathrm{height}^{2}\left(\mathrm{~kg} / \mathrm{m}^{2}\right)$. Participants were also asked to complete a questionnaire. To measure $\mathrm{UA}$ and creatinine, blood samples were collected using vacuum blood collection tubes. Participants were previously instructed not to drink or eat after 9:00 p.m. on the previous day, except for water and approved medications. Laboratory analyses of serum UA and creatinine were conducted using a Beckman Coulter, Inc. In addition, creatinine was used as an adjustment variable.

\section{Lifestyle habits}

Breslow's Health Practice Index (BHPI) was used to ascertain the level of lifestyle habits [18]. BHPI consists of 7 habits, namely amount of sleep, breakfast consumption, nutritional balance, eating between meals, alcohol consumption, tobacco smoking status, and exercise. Participants were rated as "good" or "bad" for each of the above habits based on the following criteria [19].

"Amount of sleep" comprised five categories: $4 \mathrm{~h}$ or less, from 5 to $6 \mathrm{~h}$, from 7 to $8 \mathrm{~h}$, from 9 to $10 \mathrm{~h}$, and $11 \mathrm{~h}$ or more. For responses other than "from 7 to 8 h" was coded as a bad habit. "Breakfast consumption" comprised the following three categories: daily, sometimes, and never. "Never" was coded as a bad habit. "Nutritional balance" refers to whether the subject carefully considered the nutritional balance of the food they ate. Subjects answered: always, never, and no opinion. "Never" was coded as a bad habit. "Eating between meals" comprised the following three categories: never, rarely, and almost every day. "Almost every day" was coded as a bad habit. "Alcohol consumption" comprised the following three categories: never, rarely, and every day. "Every day" was coded as a bad habit. "Smoking status" comprised the following three categories: non-smoking, every day, and smoking in the past. "Every day" was coded as a bad habit. Finally, "exercise" comprised the following three categories: regularly, sporadically, and never. "Never" was coded as a bad habit. The final score for BHPI was the total number of "bad habits"; therefore, a high score indicated an unhealthy lifestyle. In addition, BHPI was used as an adjustment variable. 
Analysis

Genetic and familial environmental factors were adjusted for using the following three methodologies: generalized linear mixed models (GLMMs), twin-pair difference value analyses using both MZ and DZ twin pairs, and twin-pair difference value analyses using MZ twin pairs only [20].

The design of GLMMs was used to control for the clustering of twins within a pair [21]. The GLMM incorporates random effects and fixed effect parameters, and random effects are usually assumed to have a normal distribution [22, 23]. Christensen et al. [24] used the following equation in their GLM model: for each twin, $i$, of a pair $(i=1,2)$ let $Y_{i}=\mathrm{UA}$ and $X_{i}=$ BMI. Let

$Y_{i}=a_{0}+a_{1} X_{i}+E_{i}$,

where $E_{i}$ represents measurement error and effects specific to twin $i$. The coefficient $a_{1}$ represents the strength of a linear association between the UA and a corresponding variable. The $a_{0}$ represents intercept.

The twin-pair difference value analyses were a relatively straightforward method. In addition, the twin-pair difference value analyses can control for all possible genetic and familial environmental factors using the different scores of $\mathrm{MZ}$ twin pairs [25]. Because MZ twin pairs share all of their genes and early life environmental factors are shared [26], if any differences in MZ twin pairs are observed in twin-pair difference value analyses, it could be inferred that there are unique environmental influences on each individual. Thus, the association between MZ difference scores of phenotypes, such as BMI and UA, suggests the presence of pure environmental associations that are not influenced by genetic and familial environmental factors.

There are three factors that affect the phenotype in twin studies: additive genetics $(A)$, common environment $(C)$, and unique environment $(E)$. This can be expressed in the following equation:

$Y_{1}=A+C+E 1$

$Y_{2}=A+C+E 2$

$Y_{1}-Y_{2}=E 1-E 2=$ unique environmental

influences on each individual

The within-pair difference is calculated as:

$D=Y_{1}-Y_{2}=a_{1} D_{x}+E$

where $D_{x}=X_{1}-X_{2}$ and $E=E_{1}-E_{2}$. From (1) it can be seen that the coefficient, $a_{1}$, can be estimated by regressing $D$ against $D_{x}$ and constraining the fitted line to pass through the origin [because (1) does not have an intercept term]. This second regression approach controls for age, sex, and genetic factors (all in MZ twins and on average, approximately half in DZ twins).
In addition, twin-pair difference value analyses utilize difference scores, and its statistical power to detect unique environmental factors is comparable to the classical twin design, which utilizes both MZ and DZ twin pairs [27]. Standardized coefficients and $95 \%$ confidence intervals were obtained by the "lme4" package [28] in R statistical software version 3.1.0 [29].

\section{Results}

Table 1 shows the number of study participants and the mean values of UA levels and other variables stratified by sex. In addition, Table 1 shows the number of BHPI variables. Items that are appended with asterisks are considered to be bad lifestyle habits.

Table 2 shows the results of analyses using GLMMs, twin-pair difference value analyses using both MZ and DZ twin pairs, and twin-pair difference value analyses using MZ twin pairs only. In the GLMMs analyses, BMI was associated with UA in men and women. In the second course of analyses using MZ and DZ twin pairs, the standardized regression coefficients increased and were still significant. In the final course of analyses, MZ twin pairs only, BMI was significantly associated with UA in men and women.

\section{Discussion}

The relationship between BMI and UA was investigated using a twin study method that can be used to remove the influence of genetic factors. The present study found that BMI was significantly associated with UA, even after adjusting for genetic and family environment factors in both men and women. In particular, when within-pair differences of BMI fluctuated by one value of standard deviation, withinpair differences of UA changed by a coefficient in the results of twin-pair difference value analysis. In addition, the values of the coefficients increased from the GLMM to twin-pair difference value analysis because twin-pair difference value analysis was adjusted for genetic and family environmental factors. Thus, the values of the coefficients of twin-pair value analysis showed the coefficients regardless of genetic and family environmental factors.

The results of the present study corroborate previous studies. In the previous studies, investigators reported that UA levels were significantly associated with BMI. Numerous epidemiological studies have shown a positive correlation between obesity and increased UA levels [30, 31]. Recently, in a Mendelian randomization analysis, it was reported that UA levels were associated with BMI, and that reduction of BMI could help improve UA levels [32]. 
Table 1 Descriptive statistics of the study sample number $(\%)$ and the mean (standard deviation)

\begin{tabular}{|c|c|c|}
\hline & $\operatorname{Men}(n=118)$ & Women $(n=280)$ \\
\hline $\operatorname{MZ}(n, \%)$ & $102(86.4 \%)$ & $262(93.6 \%)$ \\
\hline $\mathrm{DZ}(n, \%)$ & $16(13.6 \%)$ & $18(6.4 \%)$ \\
\hline Age (year, mean $\pm \mathrm{SD}$ ) & $64.10 \pm 16.28$ & $48.27 \pm 17.10$ \\
\hline BMI $\left(\mathrm{kg} / \mathrm{m}^{2}\right.$, mean $\left.\pm \mathrm{SD}\right)$ & $23.14 \pm 3.29$ & $20.93 \pm 2.47$ \\
\hline $\mathrm{UA}(\mathrm{mg} / \mathrm{dl}$, mean $\pm \mathrm{SD})$ & $5.90 \pm 1.18$ & $4.49 \pm 1.00$ \\
\hline Creatinine $(\mathrm{mg} / \mathrm{dl}$, mean $\pm \mathrm{SD})$ & $0.93 \pm 0.12$ & $0.69 \pm 0.10$ \\
\hline \multicolumn{3}{|l|}{ BHPI items } \\
\hline \multicolumn{3}{|l|}{ Amount of sleep } \\
\hline $4 \mathrm{~h}$ or $\operatorname{less}^{\mathrm{a}}(n, \%)$ & $3(2.54 \%)$ & $9(3.22 \%)$ \\
\hline $5-6 \mathrm{~h}^{\mathrm{a}}(n, \%)$ & $51(43.22 \%)$ & $146(52.14 \%)$ \\
\hline $7-8 \mathrm{~h}(n, \%)$ & $52(44.07 \%)$ & $119(42.50 \%)$ \\
\hline $9-10 \mathrm{~h}^{\mathrm{a}}(n, \%)$ & $12(10.17 \%)$ & $6(2.14 \%)$ \\
\hline 11 h or over ${ }^{\mathrm{a}}(n, \%)$ & $0(0 \%)$ & $0(0 \%)$ \\
\hline \multicolumn{3}{|l|}{ Breakfast consumption } \\
\hline Daily $(n, \%)$ & $106(89.03 \%)$ & $231(82.50 \%)$ \\
\hline Sometimes $(n, \%)$ & $5(4.24 \%)$ & $39(13.93 \%)$ \\
\hline $\operatorname{Never}^{\mathrm{a}}(n, \%)$ & $7(5.93 \%)$ & $10(3.57 \%)$ \\
\hline \multicolumn{3}{|l|}{ Nutrition balance } \\
\hline Always careful $(n, \%)$ & $67(56.78 \%)$ & $165(58.93 \%)$ \\
\hline $\operatorname{Never}^{\mathrm{a}}(n, \%)$ & $25(21.19 \%)$ & $42(15.00 \%)$ \\
\hline No opinion $(n, \%)$ & $26(22.03 \%)$ & $73(26.07 \%)$ \\
\hline \multicolumn{3}{|l|}{ Eating between meals } \\
\hline $\operatorname{Never}(n, \%)$ & $36(30.51 \%)$ & $30(10.71 \%)$ \\
\hline Rarely $(n, \%)$ & $56(47.46 \%)$ & $113(40.36 \%)$ \\
\hline Almost every day ${ }^{\mathrm{a}}(n, \%)$ & $26(22.03 \%)$ & $137(48.93 \%)$ \\
\hline \multicolumn{3}{|l|}{ Alcohol consumption } \\
\hline Never $(n, \%)$ & $36(30.51 \%)$ & $119(42.50 \%)$ \\
\hline Rarely $(n, \%)$ & $39(33.05 \%)$ & $133(47.50 \%)$ \\
\hline Every day $^{\mathrm{a}}(n, \%)$ & $43(36.44 \%)$ & $28(10.00 \%)$ \\
\hline \multicolumn{3}{|l|}{ Smoking status } \\
\hline Non-smoking $(n, \%)$ & $64(54.24 \%)$ & $251(89.64 \%)$ \\
\hline Every day $^{\mathrm{a}}(n, \%)$ & $21(17.79 \%)$ & $11(3.93 \%)$ \\
\hline Smoking in the past $(n, \%)$ & $33(27.97 \%)$ & $18(6.43 \%)$ \\
\hline \multicolumn{3}{|l|}{ Exercise } \\
\hline Regularly $(n, \%)$ & $40(33.90 \%)$ & $76(27.14 \%)$ \\
\hline Sporadically $(n, \%)$ & $42(35.59 \%)$ & $93(33.21 \%)$ \\
\hline $\operatorname{Never}^{\mathrm{a}}(n, \%)$ & $36(30.51 \%)$ & $111(39.54 \%)$ \\
\hline
\end{tabular}

$M Z$ monozygotic, DZ dizygotic, BMI body mass index, UA uric acid, BHPI Breslow's Health Practice Index

${ }^{a}$ Bad lifestyle habits
In addition, an intervention study showed significant improvements in UA levels after weight loss in obese subjects [33]. However, these studies involved the general population without taking into consideration the effect of influential genetic risk factors. Our study showed that there was a significant association between BMI and UA after removing genetic and familial environment factors. Furthermore, genome-wide association studies have indicated that UA and BMI, respectively, have specific genes which confer susceptibility [13, 15]. However, these previous studies highlight that UA and BMI, respectively, do not have common genetic risk factors i.e., there is no polygenic influence. Thus, the present study provides support to these previous studies.

In addition, we found that even if UA levels were within the normal range in women, increased UA levels while still 
Table 2 Standardized regression coefficient for the association between BMI and UA in three steps analyses

\begin{tabular}{llll}
\hline & $\begin{array}{l}\text { Individual-level analysis (GLMMs) } \\
(95 \% \mathrm{CI})\end{array}$ & $\begin{array}{l}\text { Twin-pair difference values [MZ + DZ] } \\
(95 \% \mathrm{CI})\end{array}$ & $\begin{array}{l}\text { Twin-pair difference values [MZ] } \\
(95 \% \mathrm{CI})\end{array}$ \\
\hline BMI for men & $0.194(0.016-0.373)$ & $0.382(0.145-0.620)$ & $0.333(0.072-0.594)$ \\
$\begin{array}{l}\text { BMI for } \\
\text { women }\end{array}$ & $0.186(0.071-0.302)$ & $0.272(0.112-0.432)$ & $0.314(0.151-0.477)$ \\
\hline
\end{tabular}

Adjusted standardized regression coefficient with age, creatinine, and BHPI

$B M I$ body mass index, GLMMs generalized linear mixed models, $C I$ confidence interval, $M Z$ monozygotic, DZ dizygotic, $B H P I$ Breslow's Health Practice Index

within the normal range were associated with a higher prevalence of cardiovascular risk factors [34]. Furthermore, Schretlen et al. [35] showed that UA levels at the high end of the normal spectrum were associated with an increase in white matter hyper-intensities assessed by brain MRI. White matter hyper-intensities are associated with a higher risk of stroke and dementia [36]. Therefore, it is important for health care professionals to not only encourage individuals to maintain normal UA levels, but also to prevent any increase towards higher levels within the normal range.

We speculate on the possible mechanisms, which may explain the relationship between UA and BMI. High UA has a close correlation to the mechanism of underlying renal function. Obesity and being overweight may be linked to UA levels involving two factors: excessive UA production and poor UA excretion. Among individuals with visceral fat obesity, previous studies have indicated that increased UA levels were strongly influenced by UA overproduction with a decrease in urinary urate excretion and clearance [37]. Visceral fat accumulation (VFA) induces an elevated influx of plasma free fatty acids into the hepatic portal vein and liver. This stimulates triglycerides synthesis followed by an attendant surge in UA production through the activated UA synthesis pathway [38, 39]. In addition, a number of investigators have reported that VFA is significantly associated with BMI [40, 41]. Therefore, since UA has a close biological relationship with BMI, it is important to focus on the interaction of UA and BMI for preventive medicine.

Recent studies have revealed that approximately onethird of the world's population is obese or overweight in both developed and developing countries. The increase in obese people has been particularly rapid in the past three decades [42]. In Japan, the highest age-specific prevalence of obesity and overweight defined by BMI $\geq 25 \mathrm{~kg} / \mathrm{m}^{2}$ has been observed in those who are middle-aged (aged 40-69 years) [43]. As such, adult obesity and being overweight have increased over the past few decades in Japan [44]. Therefore, one should be careful when interpreting these trends.
The strength of our study was that it used twins to adjust for genetic and familial environment factors while assessing the association between BMI and UA. However, this study has its limitations. First, because of the cross-sectional design, the direction of the causal relationship remains unclear and needs to be evaluated using large, welldesigned longitudinal studies. Second, in humans, it is generally believed that UA is the end product of purine metabolism and is produced by xanthine oxidase from xanthine, which is consequently produced from purine [45]. Furthermore, the Third National Health and Nutrition Examination Survey showed that UA levels increased with increasing consumption of purine-rich foods, such as meats, seafood, and sugar-sweetened beverages [46]. However, this study did not measure the intake of purinerich foods amongst study participants. Finally, this study did not collect information on whether participants were using medications to treat hyperuricemia.

The present study shows that there is a direct correlation between BMI and UA, after adjusting for both genetic and familial environment factors in both men and women. In future, we would like to focus on the prospective associations between BMI and UA. We have only been able to use cross-sectional studies till now; however, from this point forward, we would like to continue our research in a longitudinal format. It has been pointed out that nutritional information gathered using BHPI regarding criteria such as drinking alcohol and eating snacks is too broad, and a more detailed measure of dietary habits is necessary in order to assess its impact on BMI. Future studies will have to address this problem by developing a more comprehensive nutrition assessment index.

Acknowledgments We would like to thank all the twin pair participants who were included in the study. This work was supported by University Grants from Japanese Ministry of Education, Culture, Sports, Science and Technology (MEXT) and MEXT KAKENHI Grant Number 262931510. Laboratory tests in this research were performed courtesy of Beckman Coulter, Inc.

Conflict of interest We have no conflicts of interest to declare. 


\section{References}

1. Brand FN, McGee DL, Kannel WB, Stokes J 3rd, Castelli WP. Hyperuricemia as a risk factor of coronary heart disease: the framingham study. Am J Epidemiol. 1985;121:11-8.

2. Glynn RJ, Campion EW, Silbert JE. Trends in serum uric acid levels 1961-1980. Arthritis Rheum. 1983;26:87-93.

3. Rathmann W, Haastert B, Icks A, Giani G, Roseman JM. Tenyear change in serum uric acid and its relation to changes in other metabolic risk factors in young black and white adults: the CARDIA study. Eur J Epidemiol. 2007;22:439-45.

4. Ogura T, Matsuura K, Matsumoto Y, Mimura Y, Kishida M, Otsuka F, et al. Recent trends of hyperuricemia and obesity in Japanese male adolescents, 1991 through 2002. Metabolism. 2004;53:448-53.

5. Freedam D, Williamson D, Gunter E, Byers T. Relation of serum uric acid to mortality and ischemic heart disease. Am J Epidemiol. 1995; 141:637-44.

6. Krishman E. Hyperuricemia and incident heart failure. Circ Heart Fail. 2009;2(6):556-62.

7. Choi HK. A prescription for lifestyle change in patients with hyperuricemia and gout. Curr Opin Rheumatol. 2010;22:165-72.

8. Ezzati M, Lopez AD, Rodgers A, Vander Hoorn S, Murray CJ, Comparative Risk Assessment Collaborating Group. Selected major risk factors and global and regional burden of disease. Lancet. 2002;360:1347-60.

9. Wiseman M. The second World Cancer Research Fund/American Institute for Cancer Research expert report. Food, nutrition, physical activity, and the prevention of cancer: a global perspective. Proc Nutr Soc. 2008;67:253-6.

10. De Oliveira EP, Moreto F, Silveira LVDA, Burini RC. Dietary, anthropometric, and biochemical determinants of uric acid in free-living adults. Nutr J. 2013;12:11.

11. Kalousdian S, Fabsitz R, Havlik R, Christian J, Rosenman R. Heritability of clinical chemistries in an older twin cohort: the NHLBI Twin Study. Genet Epidemiol. 1987;4:1-11.

12. Ichida K, Matsuo H, Takada T, Nakayama A, Murakami K, Shimizu $\mathrm{T}$, et al. Decreased extra-renal urate excretion is a common cause of hyperuricemia. Nat Commun. 2012;3:764.

13. Okada Y, Sim X, Go MJ, Wu J-Y, Gu D, Takeuchi F, et al. Metaanalysis identifies multiple loci associated with kidney functionrelated traits in east Asian populations. Nat Genet. 2012;44:904-9.

14. Nan C, Guo B, Warner C, Fowler T, Barrett T, Boomsma D, et al. Heritability of body mass index in pre-adolescence, young adulthood and late adulthood. Eur J Epidemiol. 2012;27:247-53.

15. Okada Y, Kubo M, Ohmiya H, Takahashi A, Kumasaka N, Hosono N, et al. Common variants at CDKAL1 and KLF9 are associated with body mass index in east Asian populations. Nat Genet. 2012;44:302-6.

16. Hayakawa K, Iwatani Y, Osaka Twin Research Group. An overview of multidisciplinary research resources at the Osaka University Center for Twin Research. Twin Res Hum Genet. 2013;16:217-20.

17. Krenke B, Tereba A, Anderson S, Buel E, Culhane S, Finis CJ, et al. Validation of a 16-locus fluorescent multiplex system. J forensic Sci. 2002;47:773-85.

18. Belloc NB, Breslow L. Relationship of physical health and health practices status. Prev Med (Baltim). 1972;1:409-21.

19. Wada T, Fukumoto T, Ito K, Hasegawa Y, Osaki T, Ban H. Of the three classifications of healthy lifestyle habits, which one is the most closely associated with the prevention of metabolic syndrome in Japanese? Intern Med. 2009;48:647-55.

20. Carlin JB, Gurrin LC, Sterne JA, Morley R, Dwyer T. Regression models for twin studies: a critical review. Int $\mathrm{J}$ Epidemiol. 2005;34:1089-99.
21. Smith T, Smith B. PROC GENMOD with GEE to analyze correlated outcomes data using SAS. San Diego: CA Dep Def Cent; 2006. p. 1-14.

22. Breslow NE, Clayton DG. Approximate inference in generalized linear mixed models. J Am Stat Assoc. 1993;88:9-25.

23. Quirk J, Berg S, Chinchilli V, Johansson B, Mcclearn G, Vogler G. Modelling blood pressure as a continuous outcome variable in a co-twin control study. J Epidemiol Community Health. 2001;55:746-7.

24. Christensen K, Støvring H, McGue M. Do genetic factors contribute to the association between birth weight and blood pressure? J Epidemiol Community Health. 2001;55:583-7.

25. Yamagata S, Takahashi Y, Ozaki K, Fujisawa KK, Nonaka K, Ando J. Bidirectional influences between maternal parenting and children's peer problems: a longitudinal monozygotic twin difference study. Dev Sci. 2013;16:249-59.

26. Kato K, Sullivan PF, Evengard B, Pedersen NL. Chronic widespread pain and its comorbidities a population-based study. Arch Intern Med. 2006;166:1649-54.

27. Purcell S, Koenen KC. Environmental mediation and the twin design. Behav Genet. 2005;35:491-8.

28. Bates D, Maechler M, Bolker B, Walker S. lme4: linear mixedeffects models using Eigen and S4. 2014. http://cran.r-project. org/package=lme4.

29. R Core Team. R: a language and environment for statistical computing. Vienna, Austria. 2014. http://www.r-project.org/

30. Remedios C, Shah M, Bhasker AG, Lakdawala M. Hyperuricemia: a reality in the Indian obese. Obes Surg. 2012;22:945-8.

31. Liu L, Lou S, Xu K, Meng Z, Zhang Q, Song K. Relationship between lifestyle choices and hyperuricemia in Chinese men and women. Clin Rheumatol. 2013;32:233-9.

32. Palmer TM, Nordestgaard BG, Benn M, Tybjærg-Hansen A, Davey Smith G, Lawlor D, et al. Association of plasma uric acid with ischaemic heart disease and blood pressure: mendelian randomisation analysis of two large cohorts. BMJ. 2013;347:f4262.

33. Yamashita S, Matsuzawa Y, Tokunaga K, Fujioka S, Tarui S. Studies on the impaired metabolism of uric acid in obese subjects: marked reduction of renal urate excretion and its improvement by a low-calorie diet. Int J Obes. 1986;10:255-64.

34. Jin YL, Zhu T, Xu L, Zhang WS, Liu B, Jiang CQ, et al. Uric acid levels, even in the normal range, are associated with increased cardiovascular risk: the Guangzhou Biobank Cohort Study. Int J Cardiol. 2013;168:2238-41.

35. Schretlen DJ, Inscore AB, Vannorsdall TD, Kraut M, Pearlson GD, Gordon B, et al. Serum uric acid and brain ischemia in normal elderly adults. Neurology. 2007;69:1418-23.

36. Debette S, Markus HS. The clinical importance of white matter hyperintensities on brain magnetic resonance imaging: systematic review and meta-analysis. BMJ. 2010;341:c3666.

37. Matsuura F, Yamashita S, Nakamura T, Nishida M, Nozaki S, Funahashi $\mathrm{T}$, et al. Effect of visceral fat accumulation on uric acid metabolism in male obese subjects: visceral fat obesity is linked more closely to overproduction of uric acid than subcutaneous fat obesity. Metabolism. 1998;47:929-33.

38. Fox IH. Metabolic basis for disorders of purine nucleotide degradation. Metabolism. 1981;30:616-34.

39. Fabregat I, Revilla E, Machado A. Short-term control of the pentose phosphate cycle by insulin could be modulated by the nadph/nadp ratio in rat adipocytes and hepatocytes. Biochem Biophys Res Commun. 1987;146:920-5.

40. The Examination Committee of Criteria for "Obesity Disease" in Japan; Japan Society for the Study of Obesity. New criteria for 'obesity disease' in Japan. Circ J. 2002:987-92.

41. Oka R, Miura K, Sakurai M, Nakamura K, Yagi K, Miyamoto S, et al. Comparison of waist circumference with body mass index 
for predicting abdominal adipose tissue. Diabetes Res Clin Pract. 2009;83:100-5.

42. Ng M, Fleming T, Robinson M, Thomson B, Graetz N, Margono $\mathrm{C}$, et al. Global, regional, and national prevalence of overweight and obesity in children and adults during 1980-2013: a systematic analysis for the Global Burden of Disease Study 2013. Lancet. 2014;384:766-81.

43. Ministry of Health L and W. The National Health and Nutrition Survey in Japan, 2005. Daiichi Shuppan 2007.

44. Yatsuya H, Toyoshima H, Yamagishi K, Tamakoshi K, Taguri M, Harada A, et al. Body mass index and risk of stroke and myocardial infarction in a relatively lean population: meta-analysis of 16 Japanese cohorts using individual data. Circ Cardiovasc Qual Outcomes. 2010;3:498-505.

45. Suchetha Kumari N, Atul Kamath M, Sukanya Shetty, Roopa Rani Bhandary, Kathyayini. Serum uric acid as obesity related indicator in young obese adults. Res J Pharm Biol Chem Sci 2011;2:1-6.

46. Choi HK, Curhan G. Beer, liquor, and wine consumption and serum uric acid level: the Third National Health and Nutrition Examination Survey. Arthritis Rheum. 2004;51:1023-9. 